\title{
Prevalence of Low-Cost Generic Program Use in a Nationally Representative Cohort of Privately Insured Adults
}

\author{
Nathan James Pauly, BA, and Joshua David Brown, PharmD, MS
}

\begin{abstract}
BACKGROUND: Administrative claims data are used for a wide variety of research and quality assurance purposes. Despite their utility, they are prone to medication exposure misclassification if medications are purchased without utilizing an insurance benefit. Low-cost generic programs (LCGPs) offered at major chain pharmacies are a relatively new and sparsely investigated source of exposure misclassification. Since they were implemented in 2006, LCGPs are now available at 8 of the 10 largest pharmacy chains and include a wide variety of medication classes. LCGP medications are often purchased out of pocket; thus, a pharmacy claim may never be submitted and exposure may go unobserved in claims data. There are little data regarding the utilization of these programs, and estimates of their use can provide important insights into the potential impact LCGPs may have on exposure misclassification in claims data.
\end{abstract}

OBJECTIVES: To (a) quantify the prevalence of LCGP users in a privately insured adult population, (b) assess patterns of LCGP use, and (c) compare clinical and demographic characteristics associated with LCGP users and nonusers.

METHODS: The study cohort consisted of 19,037 privately insured adults aged 18-64 who participated in the Medical Expenditure Panel Survey (MEPS) from 2007-2011. MEPS captures medication utilization at the pharmacy level, so prescription fills can be observed irrespective of a claim being filed. Pharmaceutical utilization was assessed at the individual level for each year of the study period, and LCGP use was recorded as a binary variable for each individual. An LCGP medication fill was identified if the total cost of the drug was paid out of pocket and matched the cost of medications listed on LCGP formularies available from Target, Walmart, CVS, or other major pharmacy retailers during these years. Cohort demographics and characteristics of interest included age, gender, race, employment status, marital status, family income, education level, residence in a metropolitan statistical area (MSA), prescription drug coverage, geographic region, comorbidities, and number of unique medications and medication fills. Comparisons were made between users and nonusers using chisquare and t-tests. Multivariable logistic regression was used to identify factors associated with LCGP use.

RESULTS: Out of the entire study cohort $(\mathrm{N}=19,037), 6,921$ (36.4\%) individuals were identified as LCGP users, representing 34 million LCGP users annually. Users tended to be older, had higher Charlson Comorbidity Index scores, filled more prescriptions per person, and used more unique medications. Proportions of LCGP users and uses nearly doubled from 2007-2011, while total prescription utilization per person remained relatively stable. Over $10 \%$ of all prescription fills were filled through LCGPs. of all LCGP fills, approximately $42 \%$ were for cardiovascular medications, $12 \%$ for antidiabetics, and $14 \%$ for levothyroxine. Greater than $30 \%$ of fills for antigout, metronidazole, angiotensin-converting enzyme inhibitors, levothyroxine, metformin, and diuretics were obtained through LCGPs, as were $18.9 \%$ of all warfarin fills. Compared with the reference category aged 18-34, adults aged 35-54 had an adjusted odds ratio (AOR) of being an LCGP user of $1.39(95 \% \mathrm{Cl}=1.29-1.50)$ and adults aged 55-64 had an AOR of $1.86(95 \% \mathrm{Cl}=1.70-2.04)$. Additionally, those with prescription drug coverage were nearly twice as likely to be LCGP users (AOR $=1.96 ; 95 \%$ $\mathrm{Cl}=1.64-2.35$ ) compared with those without. Gender, income, comorbidity burden, region, year of panel entry, and number of unique medications also significantly predicted LCGP use.

CONCLUSIONS: There is a high rate of LCGP use in the privately insured adult population. Users of LCGPs tend to be older, have more chronic comorbidities, and use more medications than nonusers. Claims-based research and quality assurance programs focusing on the benefits and harms of medications available through these programs are at risk of greatly underestimating the true medication exposure in this population and should account for this in sensitivity analyses. Managed care organizations should incentivize the reporting of LCGP medication use or make adjustments to generic medication benefit structures to more effectively capture true medication exposure.

J Manag Care Spec Pharm. 2015;21(12):1162-70

Copyright $\odot 2015$, Academy of Managed Care Pharmacy. All rights reserved.

\section{What is already known about this subject}

- Administrative claims data maintained by managed care organizations are used for a variety of research and quality assurance purposes. A major limitation of claims data is unobserved medication use, which may result in medication exposure misclassification. Low-cost generic programs (LCGPs) have been estimated to be used by $20 \%-30 \%$ of adults with private insurance and include a variety of medication classes used to treat many common acute and chronic conditions.

When a medication is purchased through an LCGP, a claim may never be submitted and the medication use may go unobserved in administrative claims data.

\section{What this study adds}

In a nationally representative cohort of privately insured adults, this study found that $36.4 \%$ of patients filled a prescription through an LCGP and that over 10\% of all prescription fills from 2007-2011 were obtained through these programs. The proportion of privately insured adults using these programs has increased since 2007, from $21.5 \%$ up to $35.2 \%$ in 2011 .

Significant predictors of LCGP use included older age, female sex, higher comorbidity burden, having prescription drug coverage, and using multiple unique medications. 


\section{What this study adds (continued)}

Over $30 \%$ of all fills for antigout medications, metronidazole, angiotensin-converting enzyme inhibitors, levothyroxine, metformin, and diuretics were obtained through LCGPs, as were $18.9 \%$ of all warfarin fills. Of all fills through LCGPs, $42 \%$ were for cardiovascular medications, 12\% for antidiabetics, $14 \%$ for levothyroxine, $6 \%$ for contraceptives, and $5 \%$ for selective serotonin reuptake inhibitors.

$\mathrm{T}$ he usefulness and limitations of administrative claims data for drug utilization studies, pharmacovigilance, health policy research, benefit design, and quality assurance are well established. ${ }^{1-5}$ Exposure misclassification is a major limitation of administrative claims data for studies investigating the benefits or harms of medication exposure or efforts to measure adherence to medication therapy. Misclassification of drug exposure may occur when an individual acquires a medication outside of their prescription drug benefit. Common sources of misclassification include individuals not ingesting filled prescriptions, free samples provided by physicians, medications obtained but not covered under a drug benefit, paying for drugs out of pocket, and over-the-counter medications., ${ }^{1,3,6-9}$ Low-cost generic programs (LCGPs) are a sparsely investigated source of exposure misclassification.

LCGPs first appeared in late 2006, with Kmart providing 90-day supplies of certain generics for $\$ 15$ and Walmart offering 30 -day supplies for $\$ 4 .^{10}$ Since then, almost all major pharmacy chains have implemented LCGPs, including 8 of the top 10 largest chain pharmacies, which are heterogeneous regarding costs and medications included, as well as some requiring annual membership fees. ${ }^{4,10,11}$ These programs include a wide variety of medication categories (cardiovascular, antibiotics, arthritis and pain, antidiabetes, mental health, men's and women's health, etc.) and include one-third of the top 100 generics by volume used by Americans. ${ }^{12-14}$ Given the low costs associated with LCGPs, it is often cheaper for individuals to purchase LCGP drugs out of pocket than to pay a $\$ 10-\$ 20$ copay to receive the medication through a prescription drug benefit plan. ${ }^{15}$

LCGPs increase the accessibility and affordability of prescription medications, especially for individuals who lack prescription drug coverage. ${ }^{1}$ In 2008, over 70 million Americans were estimated to have used an LCGP to obtain a prescription medication-a figure that has likely expanded as the number and popularity of these programs has increased. ${ }^{10,14,16} \mathrm{~A}$ national poll conducted in 2008 found that $21 \%$ of privately insured adults reported using LCGPs. ${ }^{16}$ In contrast, a 2010 cross-sectional survey found that $31.9 \%$ of adults reported LCGP use. $^{17}$ However, these studies failed to examine the insurance characteristics of program users and the medications being obtained.
Since insurance claims may never be submitted when individuals purchase medication through LCGPs, privately insured adults who use these programs are subject to exposure misclassification in administrative claims data. Considering the uses of administrative claims data by managed care organizations, including Healthcare Effectiveness Data and Information Set (HEDIS) measures and other quality-reporting standards, ${ }^{1}$ it is prudent to fully assess the use of these programs in the insured population. This study sought to describe the trends of LCGP use in a nationally representative cohort of privately insured adults and to analyze characteristics of LCGP users and medication use attributed to these programs.

\section{Methods}

\section{Data Source}

This study relied on publicly available data from the Medical Expenditure Panel Survey (MEPS) from the years 2007-2011. MEPS is a nationally representative survey of individuals living in the United States that collects data regarding demographics and clinical conditions and healthcare and pharmaceutical utilization. MEPS uses an overlapping panel design with a new panel of participants added each year. Panels are followed for up to 2 years with 5 rounds of data collection occurring during each 2-year period. MEPS is a de-identified public use dataset supported by the Agency for Healthcare Research and Quality (AHRQ) that is intended for research purposes; therefore, it is exempt from institutional review board approval.

\section{Study Population and Design}

A cross-sectional study design over the years 2007-2011 was used to compare LCGP users and nonusers. Inclusion into the study cohort required that individuals were aged 18-64, had private insurance, participated in all 5 rounds of data collection, and reported using at least 1 prescription medication during the 2-year period. Individuals with Medicare and Medicaid or with more than one insurance type were excluded due to the potential variation in prescription drug benefits. Additionally, the Medicare drug benefit has other factors that warrant specific consideration, including the "donut hole" and coverage limits, and was deemed outside the scope of the current study. An individual's age was recorded as their age at the last round of data collection.

Pharmaceutical utilization was assessed at the individual level for each year of the study period. Pharmaceutical data in MEPS "Prescribed Medications" data files include drug name, National Drug Code (NDC), round supplied, strength, and quantity dispensed. Each prescription fill included in the MEPS dataset also includes information regarding the amount paid by the individual out of pocket and the amount contributed by other sources. Pharmacy data are collected at the pharmacy level so prescription fills are captured irrespective of whether an insurance claim was submitted. 


\section{LCGP Use}

Three stipulations were used to define LCGP use: (1) the total cost of the drug was paid out of pocket; (2) the cost of the drug exactly matched the cost of an LCGP drug as reported by pharmacies; and (3) the medication must be listed on LCGP lists available from Target, Walmart, Publix, Kmart, Walgreens, or CVS during the study period. An additional stipulation for oral medication included specific quantities dispensed for 30- and 90-day supplies of medications, with the exception of antiinfectives, steroids, and contraceptives, which were allowed to vary given differential dosing regimens for these classes. LCGP use was coded as a binary-dependent variable for any use during the study period. An individual with at least 1 LCGP use during their panel period was classified as an LCGP user and the total number of LCGP uses per person was calculated for each year of the study period.

Drug classes (e.g., angiotensin-converting enzyme inhibitors [ACEIs] and HMG-CoA reductase inhibitors [statins]) according to the Multum Lexicon classification systems (Cerner Multum, Denver, CO) were also categorized by LCGP use to accomplish 2 goals. First, it demonstrated what proportion of total LCGP uses comprised different drug classes. Second, it made it possible to determine the proportion of prescription fills in each drug class that were obtained through LCGPs.

\section{Participant Characteristics}

Cohort demographics and characteristics of interest included age, gender, race, employment status, marital status, family income, education level, residence in a metropolitan statistical area (MSA), prescription drug coverage, geographic region, presence of comorbidities, and number of unique medications. Age, employment status, marital status, income level, and MSA were all assessed at the last round of data collection from the MEPS "Full Year Consolidated" data files. The presence of comorbidities and the number of prescription fills and number of unique medications were summed for each individual over the entire study period. For comparison, the cohort was divided by age categories: 18-34, 35-54, and 55-64. Race was classified as white, Hispanic, African American, Asian, and other. Family income in MEPS is recorded as a percentage of the federal poverty level (FPL) and categorized as $<100 \%$ of FPL, $100 \%-124 \%$ of FPL, $125 \%-199 \%$ of FPL, $200 \%-399 \%$ of FPL, and $\geq 400 \%$ of FPL. Prescription drug coverage was recorded as a binary variable if an individual either reported having a usual third-party payer or was seen to have thirdparty contributions in the pharmaceutical utilization dataset. The presence of comorbidities and comorbidity burden was based on the Charlson Comorbidity Index (CCI) using the adaptation by D'hoore et al. (1996) and was recorded as the sum of the weighted score for each individual and further categorized for scores of $0-1,2-4,5-6$, and 7 or greater using the MEPS "Medical Conditions" files. ${ }^{18}$ Number of prescription fills

\section{FIGURE 1 Study Attrition}

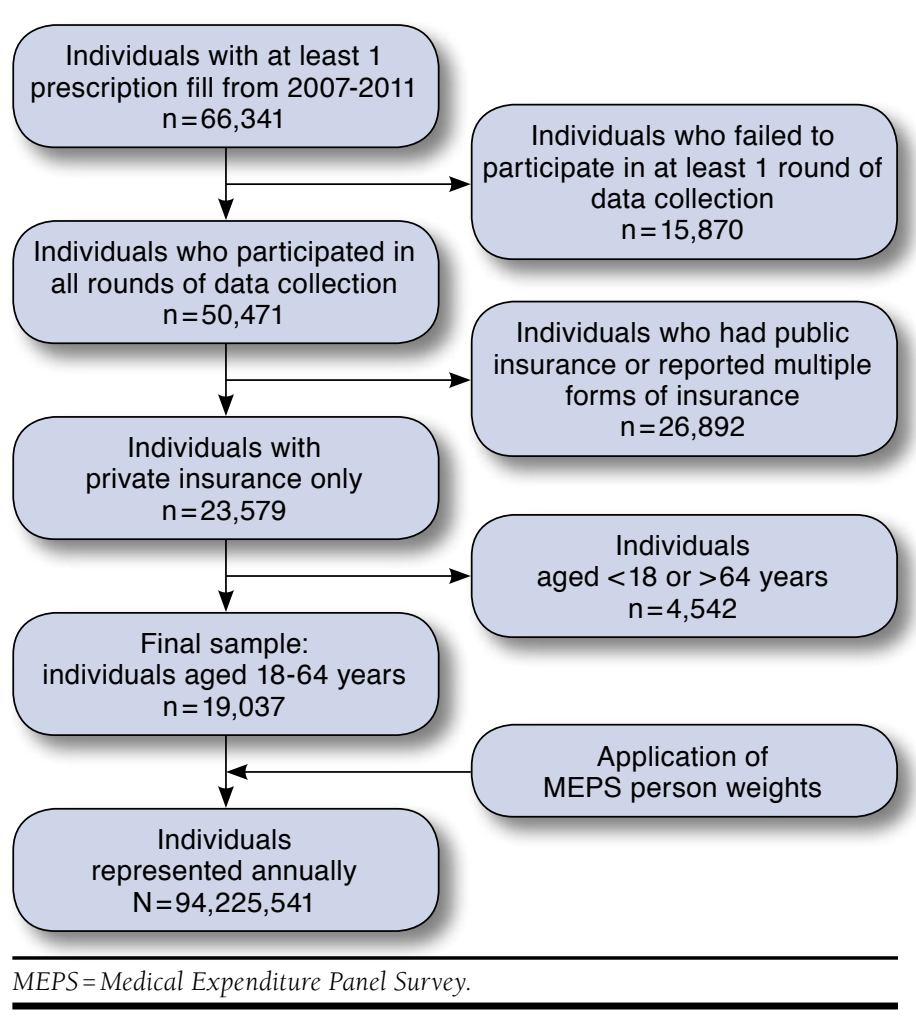

and number of unique medications were continuous measures of all prescription fills during each participant's 2-year panel period.

\section{Data Analysis}

The proportions of LCGP uses and users were tracked from 2007-the first year after LCGP implementation-to 2011the most recent year of data available from MEPS. These proportions were compared with overall pharmaceutical utilization for users and nonusers over the same time period.

Comparisons were conducted for each of the studied cohort characteristics between LCGP users and nonusers identified during the 2007 to 2011 time period using chi-square or t-tests for categorical and continuous variables, respectively. Multivariable logistic regression was used to identify factors associated with LCGP use. Adjusted odds ratios (AORs) and 95\% confidence intervals (CIs) were reported. All data analyses were conducted using SAS 9.4 (SAS Institute, Cary, NC) and implemented SAS procedures (SURVEYMEANS, SURVEYFREQ, SURVEYLOGISTIC), which take into account the complex survey design of MEPS and use the survey weights to report population estimates. This manuscript was drafted in concordance with the STROBE guidelines for reporting observational research studies. ${ }^{19}$ 


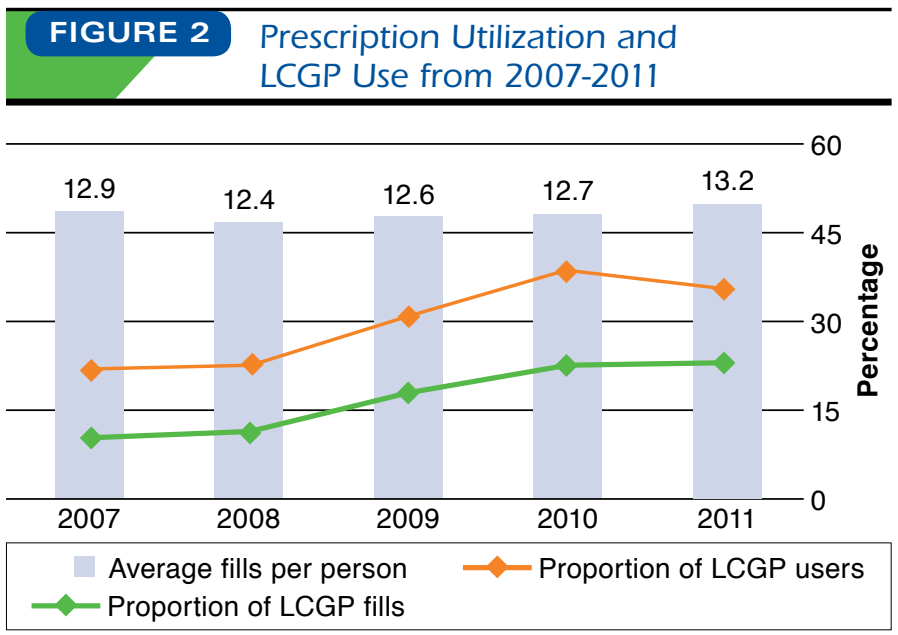

Note: Proportion of LCGP fills = number of LCGP fills/total number of fills for medications available through LCGPs.

$L C G P=$ low-cost generic program.

\section{Results}

\section{Cohort Comparisons}

A total of 19,037 privately insured individuals aged 18-64 participated in all rounds of MEPS data collection and also used at least 1 prescription medication during the study period (Figure 1). Of this population, 6,921 (36.4\%) individuals were classified as LCGP users who had at least 1 prescription fill that met the criteria for LCGP use. Using survey sampling weights in MEPS, the total cohort represents over 94 million privately insured adults and about 34 million users of LCGPs annually. The proportion of LCGP users in a calendar year increased from $21.5 \%$ of individuals in 2007 to $35.2 \%$ of individuals in 2011 (Figure 2). The trend by panel increased significantly. In the last panel of individuals surveyed over 2 years (2010-2011), 46.9\% used an LCGP medication.

User and nonuser demographic characteristics are presented in Table 1 for the study sample and for the population estimates. LCGP users tended to be older, with a greater proportion of LCGP users in the 55-64 year age group (29.6\% of users vs. $19.9 \%$ of nonusers, $P<0.001$ ) and had a median age almost 5 years greater than nonusers ( 46.9 vs. 41.8 years, data not shown). The user group also had a significantly greater proportion of individuals who were female (60.4\% vs. $52.8 \%$, $P<0.001)$, had prescription drug coverage $(98.1 \%$ vs. $95.1 \%$, $P<0.001)$, were unemployed (15.9\% vs. $13.8 \%, P<0.001$ ), and were married $(65.0 \%$ vs. $62.1 \%, P=0.008)$. Less than $10 \%$ of the nonuser group had a CCI score greater than 1 compared with the LCGP user group, where $21.2 \%$ had a CCI score greater than $1(P<0.001)$.

\section{Medication Use}

The total study cohort $(\mathrm{N}=19,037)$ filled 376,680 medications during the study period. Prescription utilization differed significantly for LCGP users and nonusers. Over the entire study period, LCGP users filled a median of 22 total prescriptions per person compared with 7 prescriptions for nonusers. LCGP users also filled nearly twice as many unique medications per person as nonusers (median 6 for users vs. 3 for nonusers). Figure 2 displays the total number of prescription fills per person for calendar years 2007-2011 graphed against the proportion of those fills that were purchased through LCGPs. Of all prescription fills in the study period $(\mathrm{N}=376,680)$, 67.1\% ( $\mathrm{n}=252,712$ ) were for medications or medication classes included on LCGP formularies from major chain pharmacies. Of those 252,712 medication fills, $16.1 \%(n=40,623)$ met the criteria for an LCGP fill. Thus LCGP fills represent $10.8 \%$ of all prescription fills, regardless of whether the medication or medication class is available through LCGPs. While the total number of fills per person remained relatively constant from 2007-2011, the proportion of prescriptions filled using LCGPs out of all drug classes available through LCGPs more than doubled from $10.2 \%$ in 2007 to $22.9 \%$ in 2011. Furthermore, LCGP uses comprised $6.8 \%$ of all prescription fills in 2007 and $15.6 \%$ of all fills in 2011 .

Figure 3 shows medications and medication classes that are available through LCGPs and the proportion of these categories that were filled via LCGPs. Greater than 30\% of fills for antigout medications, metronidazole, ACE inhibitors, levothyroxine, metformin, and diuretics met the criteria for LCGP fills. Between $20 \%$ and $30 \%$ of prescriptions for tricyclic antidepressants, sulfonylureas, steroids, beta blockers, and anti-infectives (antifungals, cephalosporins, penicillins, fluoroquinolones, sulfamethoxazole-trimethoprim, tetracyclines, and topical anti-infectives) also met the criteria for LCGP fills. Of all prescriptions obtained through LCGPs, approximately $42 \%$ were for cardiovascular drugs (ACE inhibitors, statins, angiotensinreceptor blockers, calcium channel blockers, nitroglycerin, beta blockers, alpha antagonists, diuretics, antiarrhythmics), $14 \%$ for levothyroxine, $12 \%$ for antidiabetic medications (metformin and sulfonylureas), $6 \%$ for contraceptives, and 5\% for selective serotonin reuptake inhibitors (Figure 4).

\section{Determinants of LCGP Use}

Cohort demographics and dummy variables for each study panel and the number of unique medications used were included in a multivariable logistic regression model. After adjusting for all covariates, age, gender, prescription drug coverage, income category, CCI score, region, panel year, and number of unique medications all significantly predicted LCGP use. Odds ratios and 95\% CIs for the significant variables are presented in Table 2. 
Prevalence of Low-Cost Generic Program Use in a Nationally Representative Cohort of Privately Insured Adults

TABLE 1 Demographic Characteristics of Study Cohort

\begin{tabular}{|c|c|c|c|c|c|c|c|c|}
\hline \multirow[b]{2}{*}{$\begin{array}{l}\text { Overall sample: } N=19,037 \\
\text { Population estimate: } N=94,225,541\end{array}$} & \multicolumn{4}{|c|}{ LCGP Users } & \multicolumn{4}{|c|}{ Nonusers } \\
\hline & $\mathrm{N}$ & $\%$ & $\begin{array}{c}\mathrm{N} \\
\text { (Weighted) }\end{array}$ & $\begin{array}{c}\% \\
\text { (Weighted) }\end{array}$ & $\mathrm{N}$ & $\%$ & $\begin{array}{c}\mathrm{N} \\
\text { (Weighted) }\end{array}$ & $\begin{array}{c}\% \\
\text { (Weighted) } \\
\end{array}$ \\
\hline & 6,921 & 36.4 & $34,345,073$ & 36.4 & 12,116 & 63.6 & $59,880,468$ & 63.6 \\
\hline \multicolumn{9}{|l|}{ Age $^{a}$} \\
\hline $18-34$ & 1,447 & 20.9 & $7,684,210$ & 22.4 & 3,705 & 30.6 & $19,303,791$ & 32.2 \\
\hline $35-54$ & 3,441 & 49.7 & $16,510,548$ & 48.1 & 5,995 & 49.5 & $28,639,007$ & 47.8 \\
\hline $55-64$ & 2,033 & 29.4 & $10,150,315$ & 29.6 & 2,416 & 19.9 & $11,937,670$ & 19.9 \\
\hline \multicolumn{9}{|l|}{ Gender $^{\mathrm{a}}$} \\
\hline Male & 2,646 & 38.2 & $13,625,009$ & 39.7 & 5,533 & 45.7 & $28,263,462$ & 47.2 \\
\hline Female & 4,275 & 61.8 & $20,720,064$ & 60.4 & 6,583 & 54.3 & $31,617,006$ & 52.8 \\
\hline \multicolumn{9}{|l|}{ Insurance ${ }^{a}$} \\
\hline Prescription coverage & 6,760 & 97.7 & $33,653,053$ & 98.1 & 11,485 & 94.8 & $56,986,168$ & 95.1 \\
\hline No prescription coverage & 161 & 2.3 & 692,020 & 2.0 & 631 & 5.2 & $2,894,300$ & 4.8 \\
\hline \multicolumn{9}{|l|}{ Employment $^{\mathrm{a}}$} \\
\hline Employed & 5,782 & 83.5 & $28,926,177$ & 84.3 & 10,408 & 85.9 & $51,639,421$ & 86.2 \\
\hline Unemployed & 1,139 & 16.5 & $5,418,896$ & 15.8 & 1,708 & 14.1 & $8,241,047$ & 13.8 \\
\hline \multicolumn{9}{|l|}{ Education } \\
\hline High school or less & 3,899 & 56.3 & $17,941,090$ & 52.3 & 6,858 & 56.6 & $31,787,127$ & 53.0 \\
\hline Greater than high school & 3,022 & 43.7 & $16,403,983$ & 47.8 & 5,258 & 43.4 & $28,093,341$ & 46.9 \\
\hline \multicolumn{9}{|l|}{ Marital status $^{\mathrm{a}}$} \\
\hline Married & 4,540 & 65.6 & $22,299,967$ & 65.0 & 7,714 & 63.7 & $37,203,265$ & 62.1 \\
\hline Not married & 2,381 & 34.4 & $12,045,106$ & 35.0 & 4,402 & 36.3 & $22,677,203$ & 37.9 \\
\hline \multicolumn{9}{|l|}{ Income category ${ }^{a}$} \\
\hline$<100 \%$ of FPL & 231 & 3.3 & $1,038,187$ & 3.0 & 472 & 3.9 & $1,905,426$ & 3.2 \\
\hline $100 \%-125 \%$ of FPL & 167 & 2.4 & 577,118 & 1.7 & 296 & 2.4 & $1,022,017$ & 1.7 \\
\hline $125 \%-200 \%$ of FPL & 690 & 10.0 & $2,700,288$ & 7.9 & 1,187 & 9.8 & $4,564,646$ & 7.6 \\
\hline $200 \%-400 \%$ of FPL & 2,498 & 36.1 & $11,576,753$ & 33.8 & 4,063 & 33.5 & $18,366,940$ & 30.6 \\
\hline$>400 \%$ of FPL & 3,335 & 48.2 & $18,452,726$ & 53.8 & 6,098 & 50.3 & $34,021,439$ & 56.8 \\
\hline \multicolumn{9}{|l|}{ CCI score ${ }^{a}$} \\
\hline$\leq 1$ & 5,400 & 78.0 & $27,091,549$ & 79.0 & 10,932 & 90.2 & $54,389,466$ & 90.8 \\
\hline $2-4$ & 1,446 & 20.9 & $6,859,445$ & 20.0 & 1,136 & 9.4 & $5,255,871$ & 8.8 \\
\hline $5-6$ & 57 & 0.8 & 294,891 & 0.9 & 32 & 0.3 & 142,708 & 0.2 \\
\hline$\geq 7$ & 18 & 0.3 & 99,189 & 0.3 & 16 & 0.1 & 92,422 & 0.2 \\
\hline \multicolumn{9}{|l|}{ Race $^{a}$} \\
\hline White & 4,261 & 61.6 & $26,701,056$ & 77.9 & 7,374 & 60.9 & $45,472,135$ & 75.9 \\
\hline Hispanic & 988 & 14.3 & $2,685,980$ & 7.8 & 1,804 & 14.9 & $5,064,367$ & 8.5 \\
\hline Black & 1,099 & 15.9 & $3,086,388$ & 9.0 & 1,747 & 14.4 & $5,360,882$ & 8.9 \\
\hline Asian & 398 & 5.8 & $1,167,003$ & 3.4 & 895 & 7.4 & $2,793,241$ & 4.7 \\
\hline Other & 175 & 2.5 & 704,645 & 2.1 & 296 & 2.4 & $1,189,842$ & 2.0 \\
\hline \multicolumn{9}{|l|}{ MSA } \\
\hline Rural & 976 & 14.1 & $5,322,177$ & 15.5 & 1,643 & 13.6 & $8,758,959$ & 14.6 \\
\hline Urban & 5,945 & 85.9 & $29,022,896$ & 84.5 & 10,473 & 86.4 & $51,121,509$ & 85.4 \\
\hline \multicolumn{9}{|l|}{ Region $^{\mathrm{a}}$} \\
\hline Northeast & 1,005 & 14.5 & $5,991,511$ & 17.5 & 2,026 & 16.7 & $12,178,151$ & 20.3 \\
\hline Midwest & 1,597 & 23.1 & $7,976,208$ & 23.3 & 2,869 & 23.7 & $14,152,932$ & 23.6 \\
\hline South & 2,614 & 37.8 & $13,078,409$ & 38.1 & 4,222 & 34.9 & $20,786,352$ & 34.7 \\
\hline West & 1,705 & 24.6 & $7,298,945$ & 21.3 & 2,999 & 24.8 & $12,763,033$ & 21.3 \\
\hline \multicolumn{9}{|l|}{ Panel year } \\
\hline 2007 & 760 & 21.2 & $18,876,188$ & 22.5 & 2,822 & 78.8 & $65,024,676$ & 77.5 \\
\hline 2008 & 1,205 & 34.1 & $29,905,406$ & 30.9 & 2,333 & 65.9 & $66,787,667$ & 69.1 \\
\hline 2009 & 1,470 & 34.0 & $36,471,999$ & 37.2 & 2,851 & 65.9 & $61,470,612$ & 62.8 \\
\hline 2010 & 1,665 & 42.2 & $41,295,165$ & 42.9 & 2,276 & 57.8 & $54,911,671$ & 57.1 \\
\hline 2011 & 1,821 & 49.8 & $45,176,609$ & 46.9 & 1,834 & 50.2 & $51,207,714$ & 53.1 \\
\hline \multicolumn{9}{|l|}{ Total number of medication fills $\mathrm{s}^{\mathrm{a}}$} \\
\hline Median (IQR) & 22 & $(9-47)$ & 22.7 & $(8.9-47.3)$ & 7 & $(2-20)$ & 6.9 & $(1.9-19.4)$ \\
\hline Unique medications used ${ }^{a}$ & & & & & & & & \\
\hline Median (IQR) & 6 & $(3-10)$ & 5.6 & $(3.0-9.5)$ & 3 & $(2-5)$ & 2.5 & $(1.1-5.0)$ \\
\hline
\end{tabular}

a $<<0.05$ between-group comparison; percentages that do not sum to $100 \%$ are due to rounding errors.

$C C I=$ Charlson Comorbidity Index; FPL =federal poverty level; $I Q R=$ interquartile range; $L C G P=l o w$-cost generic program; MSA = metropolitan statistical area . 
FIGURE 3 Proportion of Total Fills for Each Medication Class Filled Through LCGPs

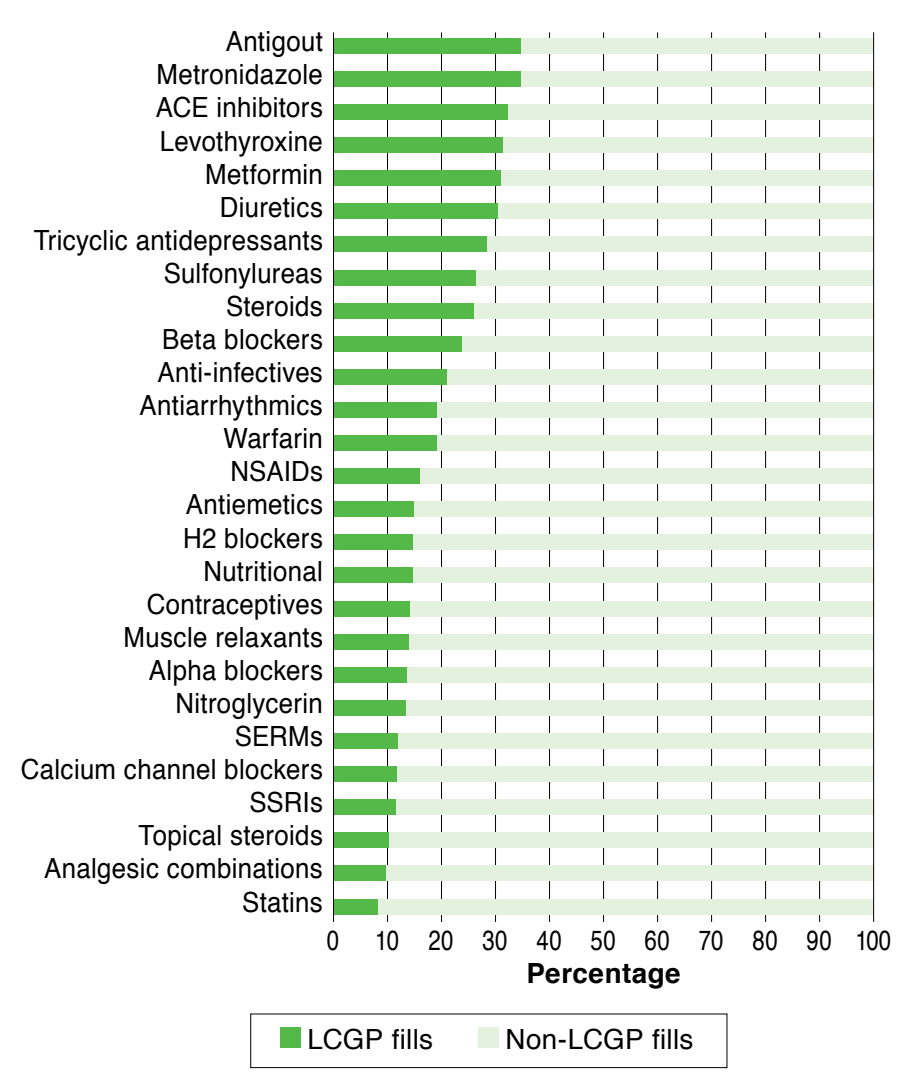

Note: Proportion of fills for each medication class through an LCGP divided by the total number of fills for the medication class.

$A C E=$ angiotensin-converting enzyme; $L C G P=$ low-cost generic program;

NSAID = nonsteroidal anti-inflammatory drug; SERM = selective estrogen receptor modulator; SSRI = selective serotonin reuptake inhibitor.

Older age was a significant predictor of LCGP use. Compared with the reference category aged 18-34, adults aged 35-54 were 39\% more likely to be LCGP users (95\% CI $=1.29-1.50)$ and adults aged 55-64 were $86 \%$ more likely to be users (95\% CI $=1.70-2.04)$. Females were significantly more likely than males to use LCGPs (AOR=1.42 [95\% CI=1.33-1.51]). Compared with individuals without prescription drug coverage, adults with prescription drug benefits were 96\% more likely to be LCGP users (95\% CI = 1.64-2.35). Compared with individuals with CCI scores $\leq 1$, those with CCI scores from 2-4 had increased odds of being LCGP users (AOR $=2.29$ [95\% CI $=2.10-2.51]$ ), as did those with CCI scores from 5-6 $(\mathrm{AOR}=2.79 \quad[95 \% \mathrm{CI}=1.79-4.36])$; however, no significant difference was observed for individuals with a CCI score $\geq 7$. Each additional unique medication used increased the odds of LCGP use by 18\% (95\% CI=1.17-1.19). Individuals resid- ing in the southern $(\mathrm{AOR}=1.28[95 \% \mathrm{CI}=1.17-1.41])$, western $(\mathrm{AOR}=1.19[95 \% \mathrm{CI}=1.07-1.31])$, or midwestern $(\mathrm{AOR}=1.20$ [95\% CI $=1.08-1.33]$ ) regions of the United States were significantly more likely to use LCGPs compared with those in the northern region reference category. Significant findings for participants beginning their panels from 2007-2010 were observed, showing increased use relative to individuals in the 2006-2007 panel. The overall model c-statistic was 0.741 , showing good model discrimination between users and nonusers.

\section{Discussion}

Our study found that more than 1 in 3 privately insured adults filled a prescription through an LCGP during the 2007-2011 study period. While the proportion of LCGP medication fills and LCGP users increased dramatically over the study period, the number of total prescription fills per person per year remained relatively stable. Thus, the increasing use of LCGPs cannot be attributed to greater pharmaceutical utilization in general-rather, it is likely due to expansion in the number of LCGPs available, an increase in the number of medications offered, and an increase in popularity and knowledge of these programs.

Our results confirm the findings of several previous studies. One study used laboratory monitoring claims as a surrogate for warfarin use. ${ }^{20}$ This study determined that the percentage of warfarin fills that failed to appear in an administrative claims dataset increased from $8.4 \%$ in 2003 to $10.5 \%$ in 2007 and up to $12.8 \%$ in $2009 .{ }^{20}$ Our study confirmed this trend but found a much greater increase in potentially misclassified warfarin fills. In our findings, $10.8 \%$ of warfarin fills met the criteria for LCGP fills in 2007, which increased to $16.5 \%$ in 2009. Over our entire study period, which also included medication use in 2010 and 2011, 314 out of 1,657 (18.9\%) warfarin fills met the criteria for LCGP use. Assuming LCGP use is indicative of exposure misclassification, this comparison shows that our definition of LCGP use was robust and consistent when compared with other studies that used surrogate indicators of medication use.

Another study examined the prevalence of LCGP use in a privately insured adult population and found that $21 \%$ of privately insured adults reported LCGP use in $2007 .{ }^{16} \mathrm{~A}$ different cross-sectional survey reported that the prevalence of users in 2010 was $31.9 \% .{ }^{17}$ However, these studies suffered from several important shortcomings that were avoided in our study design. The 2008 survey relied on a national poll; however, they do not present summary statistics for the study cohort nor do they investigate specific medication use or demographic differences between users and nonusers. ${ }^{16}$ Similarly, the 2011 survey relied on a convenience sample of only 163 individuals and failed to differentiate participants based on insurance status. ${ }^{17}$ Our study presents a more robust estimate of LCGP use because it avoids these limitations and relies on a large, nationally 


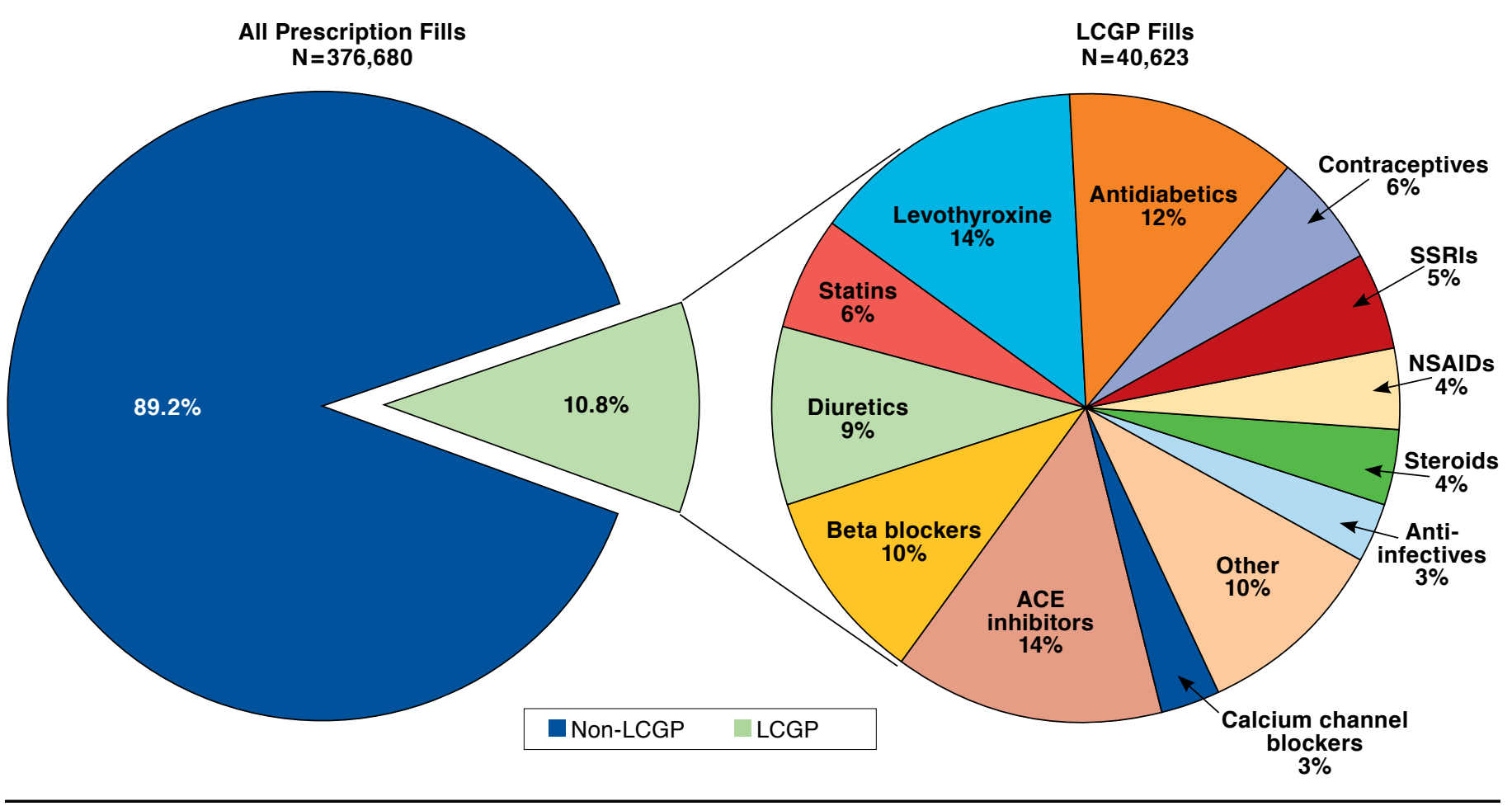

ACE= angiotensin-converting enzyme; LCGP = low-cost generic program; NSAID = nonsteroidal anti-inflammatory drug; SSRI=selective serotonin reuptake inhibitor.

representative sample and provides a complete picture of medication use in the population.

We chose MEPS as a data source as it gathers information directly from participants' pharmacies for all medications regardless of a claim being submitted to a third party. MEPS also includes payment information from all sources, including out-of-pocket payment and payment from third parties, and removes the chance of recall bias typical of most surveys of medication use. ${ }^{21}$ MEPS additionally allows for population estimates based on the sampling weights provided. Thus, our results are generalizable for the entire privately insured adult population.

Medication use in this population included classes with significant risk profiles or medications used to treat serious medical conditions. Much attention is given to adherence to antihypertensive and oral antidiabetic medications as quality metrics of health insurance plans. Based on our findings, a high proportion of uses of each of these classes may go unobserved, which could have a tremendous impact on adherence or health outcomes studies that rely on administrative claims to measure medication utilization. Additionally, in the case of warfarin, anticonvulsants, and other medications with narrow therapeutic windows, these medications have the potential to contribute significant adverse outcomes and require close monitoring for safety and efficacy or for important drug-drug or drug-disease interactions to go unnoticed in automated safety alert systems. For studies that attempt to quantify the risk of these medications, our results show that a large number of uses may go unaccounted for. Thus, the true risk may be underestimated, as a misclassification of harmful medication exposure will most often bias the association between medication use and medication harm toward the null. ${ }^{22-24}$ These results can be used by researchers to gauge the magnitude of exposure misclassification that may be present in claims studies due to LCGPs and account for these estimates in sensitivity analyses. These measures may be even more important in studies investigating medication use in subgroups found to use LCGPs more frequently, including females, older individuals, and persons who utilize more medications.

From a patient perspective, low-cost generics increase access to affordable medications. ${ }^{4,14,25}$ Especially for those without insurance coverage, LCGPs may be the only source to effective medications. Although insured individuals also stand to benefit from LCGPs, these programs introduce the chance that the added protections (i.e. surveillance for drug interactions, etc.) provided by the claims-adjudication process may 


\begin{tabular}{|c|c|c|c|}
\hline $\begin{aligned} \text { TABLE 2 } & \text { Mult } \\
& \text { Resu } \\
& \text { for L }\end{aligned}$ & \multicolumn{3}{|c|}{$\begin{array}{l}\text { Multivariable Logistic Regression } \\
\text { Results of Predictive Characteristics } \\
\text { for LCGP Use }\end{array}$} \\
\hline \multirow{2}{*}{$\frac{\text { Significant Variables }}{\text { Age }}$} & \multirow[t]{2}{*}{$\begin{array}{c}\text { Adjusted } \\
\text { Odds Ratio }\end{array}$} & \multicolumn{2}{|c|}{$\begin{array}{c}95 \% \text { Wald } \\
\text { Confidence Limits }\end{array}$} \\
\hline & & Lower & Upper \\
\hline $18-34$ & Ref. & Ref. & Ref. \\
\hline $35-54$ & 1.39 & 1.29 & 1.50 \\
\hline $55-64$ & 1.86 & 1.70 & 2.04 \\
\hline \multicolumn{4}{|l|}{ Gender } \\
\hline Male & Ref. & Ref. & Ref. \\
\hline Female & 1.42 & 1.33 & 1.51 \\
\hline \multicolumn{4}{|l|}{ Insurance coverage } \\
\hline No prescription coverage & Ref. & Ref. & Ref. \\
\hline Prescription coverage & 1.96 & 1.64 & 2.35 \\
\hline \multicolumn{4}{|l|}{ Income category } \\
\hline$<100 \%$ of FPL & Ref. & Ref. & Ref. \\
\hline $100 \%-125 \%$ of FPL & 1.19 & 0.92 & 1.53 \\
\hline $125 \%-200 \%$ of FPL & 1.20 & 0.99 & 1.45 \\
\hline $200 \%-400 \%$ of FPL & 1.24 & 1.04 & 1.47 \\
\hline$>400 \%$ of FPL & 1.09 & 0.92 & 1.30 \\
\hline \multicolumn{4}{|l|}{ CCI score } \\
\hline$\leq 1$ & Ref. & Ref. & Ref. \\
\hline $2-4$ & 2.29 & 2.10 & 2.51 \\
\hline $5-6$ & 2.79 & 1.79 & 4.36 \\
\hline$\geq 7$ & 1.74 & 0.87 & 3.47 \\
\hline \multicolumn{4}{|l|}{ Region } \\
\hline Northeast & Ref. & Ref. & Ref. \\
\hline Midwest & 1.20 & 1.08 & 1.33 \\
\hline South & 1.28 & 1.17 & 1.41 \\
\hline West & 1.19 & 1.07 & 1.31 \\
\hline \multicolumn{4}{|l|}{ Panel years } \\
\hline 2007 & Ref. & Ref. & Ref. \\
\hline 2008 & 1.54 & 1.38 & 1.72 \\
\hline 2009 & 2.12 & 1.92 & 2.35 \\
\hline 2010 & 2.87 & 2.59 & 3.17 \\
\hline 2011 & 3.05 & 2.75 & 3.39 \\
\hline Number of unique meds & 1.18 & 1.17 & 1.19 \\
\hline \multicolumn{4}{|c|}{$\begin{array}{l}\text { Note: Insignificant variables: employment, race, education, marital status, MSA } \\
C C I=\text { Charlson Comorbidity Index; } F P L=\text { federal poverty level; } L C G P=l o w \text {-cost } \\
\text { generic program; } M S A=\text { metropolitan statistical area. }\end{array}$} \\
\hline
\end{tabular}

be circumvented. Likewise, true medication utilization will not be captured in the administrative claims for privately insured individuals who use LCGPs, which may affect the findings of health services research using these data sources. ${ }^{9}$

There are several ways to mitigate the potential for exposure misclassification due to LCGPs. If pharmacists file a claim even when patients pay entirely out of pocket, then LCGP use will appear in administrative claims data as a zero-paid claim. When a medication is purchased through an LCGP, pharmacists have no reason to file claims for customers paying out of pocket. Unless commercial insurance companies are able to incentivize this activity in some way, such as covering the
LCGP payment as the "usual and customary" price, some information will inevitably be missing from claims datasets. Thus, efforts to address quality assurance and pharmacovigilance should supplement administrative claims research with data from alternate sources. Data sources that are not susceptible to missing claims include publicly available research data such as MEPS or proprietary datasets, which capture claims at the pharmacy level. Future research should compare the exposure profiles of different data sources to determine the completeness of available data and show empiric examples of how this source of exposure misclassification can affect claims-based studies.

\section{Limitations}

Our study is subject to several limitations. It may remain possible that not all medication use is recorded if all pharmacies used were not surveyed and because over-the-counter medication is not captured in MEPS. Our study definition of LCGP use may allow for overestimation of use if only pricing is considered. However, this effect is mitigated by requiring specific quantities supplied for oral medications. We chose to focus on privately insured, nonelderly adults as an example of how LCGPs could affect medication utilization in administrative claims data. Individuals with Medicaid insurance would likely have variable utilization, as Medicaid benefits differ from state to state. Likewise, LCGP use among Medicare beneficiaries may also depend on a variety of other unique factors including plan characteristics and the Part D coverage gap, which warrant specific attention. Purchasing medications out of pocket is a wellknown source of exposure misclassification in administrative claims data. This study relies on the fundamental assumption that medications purchased through LCGPs will be paid for out of pocket and thus will be unobserved in claims data.

\section{Conclusions}

This study found a high proportion of LCGP users in a nationally representative sample of privately insured adults. Additionally, multiple medication classes used in quality metrics and those with potentially serious effects and narrow therapeutic indices were found to have a high proportion of fills through these programs. Considering the implications for patients and the healthcare system as a whole, managed care organizations should facilitate the reporting of LCGP medication fills so that medication use data are captured and accounted for in quality assurance programs and pharmacoepidemiological research. Future studies should empirically demonstrate the effects of LCGP-related exposure misclassification on the results of claims-based study and the implications for quality assurance. 


\section{Authors}

NATHAN JAMES PAULY, BA, is a Graduate Student and Research Assistant, and JOSHUA DAVID BROWN, PharmD, MS, is the Humana-Pfizer Research Fellow, Institute for Pharmaceutical Outcomes and Policy, Department of Pharmacy Practice and Science, University of Kentucky College of Pharmacy, Lexington.

AUTHOR CORRESPONDENCE: Joshua David Brown, PharmD, MS, University of Kentucky College of Pharmacy, 789 S. Limestone, Lexington, KY 40506. Tel.: 479.650.8047; Fax: 859.323.0069;

E-mail: josh.brown@uky.edu.

\section{DISCLOSURES}

No sources of funding were used to conduct this study, and no input or guidance for the design, methods, or drafting of the manuscript were provided by any company or corporation. The authors have no financial disclosures or conflicts of interest.

Both authors contributed equally to data collection and to the writing and revision of the manuscript. Study concept and design and data interpretation were primarily contributed by Brown, along with Pauly.

\section{REFERENCES}

1. Schneeweiss S, Avorn J. A review of uses of health care utilization databases for epidemiologic research on therapeutics. J Clin Epidemiol. 2005;58(4):323-37.

2. Zhan C, Miller MR. Administrative data based patient safety research: a critical review. Qual Saf Health Care. 2003;12( Suppl 2):ii58-63.

3. Gavrielov-Yusim N, Friger M. Use of administrative medical databases in population-based research. J Epidemiol Community Health. 2014;68(3):283-87.

4. Choudhry NK, Shrank WH. Four-dollar generics-increased accessibility, impaired quality assurance. N Engl J Med. 2010;363(20):1885-87.

5. Willke R, Mullins, D. "Ten Commandments" for conducting comparative effectiveness research using "real-world data." J Manag Care Pharm. 2011;17

(9 Suppl A):S10-S15. Available at: http://amcp.org/WorkArea/

DownloadAsset.aspx?id=13714.

6. Gamble JM, McAlister FA, Johnson JA, Eurich DT. Restrictive drug coverage policies can induce substantial drug exposure misclassification in pharmacoepidemiologic studies. Clin Ther. 2012;34(6):1379-86.e3.

7. Li X, Sturmer T, Brookhart MA. Evidence of sample use among new users of statins: implications for pharmacoepidemiology. Med Care. 2014;52(9):773-80

8. Polinski JM, Schneeweiss S, Levin R, Shrank WH. Completeness of retail pharmacy claims data: implications for pharmacoepidemiologic studies and pharmacy practice in elderly patients. Clin Ther. 2009;31(9):2048-59.

9. Jacobus S, Schneeweiss S, Chan KA. Exposure misclassification as a result of free sample drug utilization in automated claims databases and its effect on a pharmacoepidemiology study of selective COX-2 inhibitors. Pharmacoepidemiol Drug Saf. 2004;13(10):695-702.
10. Czechowski JL, Tjia J, Triller DM. Deeply discounted medications: implications of generic prescription drug wars. J Am Pharm Assoc (2003). 2010;50(6):752-57.

11. Patel HK, Dwibedi ND, Omojasola A, Sansgiry SS. Impact of generic drug discount programs on managed care organizations. Am J Pharm Ben 2011;3(1):45-53. Available at: http://www.ajmc.com/journals/ajpb/2011/ ajpb_1ljan/ajpb_1ljan_patel_generic_45to53. Accessed October 21, 2015.

12. Walmart. Retail prescription program drug list. August 1, 2014. Available at: http://i.walmartimages.com/i/if/hmp/fusion/customer_list.pdf. Accessed October 12, 2015.

13. Walgreens. Prescription Savings Club. Value-priced medication list. August 1, 2014. Available at: http://www.walgreens.com/images/psc/VPG_ List_Update_7-25-2014.pdf. Accessed October 12, 2015.

14. Rucker NL. $\$ 4$ generics: how low, how broad, and why patient engagement is priceless. J Am Pharm Assoc. 2010;50(6):761-63.

15. Tungol A, Starner CI, Gunderson BW, Schafer JA, Qiu Y, Gleason PP. Generic drug discount programs: are prescriptions being submitted for pharmacy benefit adjudication? J Manag Care Pharm. 2012;18(9):690700. Available at: http://www.amcp.org/WorkArea/DownloadAsset. aspx?id=15873.

16. C.S. Mott Children's Hospital. Nearly 70 million Americans using discount generic rx programs. February 11, 2008. Available at: http:// mottnpch.org/sites/default/files/documents/021108GenericRxPrograms.pdf. Accessed October 13, 2015.

17. Gatwood J, Tungol A, Truong C, Kucukarslan SN, Erickson SR. Prevalence and predictors of utilization of community pharmacy generic drug discount programs. J Manag Care Pharm. 2011;17(6):449-55. Available at: http://www.amcp.org/WorkArea/DownloadAsset.aspx?id=10466.

18. D'Hoore W, Bouckaert A, Tilquin C. Practical considerations on the use of the Charlson comorbidity index with administrative databases. J Clin Epidemiol. 1996;49(12):1429-33.

19. von Elm E, Altman DG, Egger M, Pocock SJ, Gøtzsche PC, Vandenbroucke JP. The Strengthening the Reporting of Observational Studies in Epidemiology (STROBE) Statement: guidelines for reporting observational studies. Ann Intern Med. 2007;147(8):573-77.

20. Lauffenburger JC, Balasubramanian A, Farley JF, et al. Completeness of prescription information in US commercial claims databases. Pharmacoepidemiol Drug Saf. 2013;22(8):899-906.

21. Lasky T. Estimates of pediatric medication use in the United States: current abilities and limitations. Clin Ther. 2009;31(2):436-45.

22. Jurek AM, Greenland S, Maldonado G. How far from non-differential does exposure or disease misclassification have to be to bias measures of association away from the null? Int J Epidemiol. 2008;37(2):382-85.

23. Blair A, Stewart P, Lubin JH, Forastiere F. Methodological issues regarding confounding and exposure misclassification in epidemiological studies of occupational exposures. Am J Ind Med. 2007;50(3):199-207.

24. Wacholder S, Hartge P, Lubin JH, Dosemeci M. Non-differential misclassification and bias towards the null: a clarification. Occup Environ Med. 1995;52(8):557-58.

25. Zhang Y, Zhou L, Gellad WF. Potential savings from greater use of $\$ 4$ generic drugs. Arch Intern Med. 2011;171(5):468-69. 